\title{
Quasilinear problems with the competition between convex and concave nonlinearities and variable potentials
}

\author{
Dragos-Patru Covei \\ Constantin Brancusi University of Tg-Jiu, Calea Eroilor, No 30, Tg-Jiu, Gorj, Romania \\ E-mail: patrucovei@yahoo.com
}

\begin{abstract}
The purpose of this paper is to prove some existence and non-existence theorems for the nonlinear elliptic problems of the form $-\Delta_{p} u=\lambda k(x) u^{q} \pm h(x) u^{\sigma}$ if $x \in \Omega$, subject to the Dirichlet conditions $u_{1}=u_{2}=0$ on $\partial \Omega$. In the proofs of our results we use the subsuper solutions method and variational arguments. Related results as obtained here have been established in [Z. Guo and Z. Zhang, $W^{1, p}$ versus $C^{1}$ local minimizers and multiplicity results for quasilinear elliptic equations, Journal of Mathematical Analysis and Applications, Volume 286, Issue 1, Pages 32-50, 1 October 2003.] for the case $k(x)=h(x)=1$. Our results reveal some interesting behavior of the solutions due to the interaction between convex-concave nonlinearities and variable potentials.
\end{abstract}

2000 Mathematics Subject Classification: 35J60;35J20;35A05.

Key words: Bifurcation problem; Anisotropic continuous media; Existence; Non-existence.

\section{Introduction and the main results}

In this article we study the existence and non-existence of solutions for the quasilinear elliptic problems $\left(P_{\lambda}\right)_{ \pm}$of the following type

$$
-\Delta_{p} u=\lambda k(x) u^{q} \pm h(x) u^{\sigma} \text { if } x \in \Omega, u>0 \text { in } \Omega, u=0 \text { of } \partial \Omega \quad\left(\left(P_{\lambda}\right)_{ \pm}\right)
$$

where $\lambda$ is a positive real parameter, $\Omega \subset \mathbb{R}^{N}(N \geq 2)$ is a bounded domain with smooth boundary, $0<q<p-1<\sigma$, the variable weight functions $k, h \in L^{\infty}(\Omega)$ satisfy ess $\inf _{x \in \Omega} k(x)>0$ and essinf $\operatorname{in}_{x \in \Omega} h(x)>0$, and $\Delta_{p} u:=\operatorname{div}\left(|\nabla u|^{p-2} \nabla u\right), 1<p<\infty$ stands for the p-Laplacian operator.

We call a function $u: \Omega \rightarrow \mathbb{R}$ a solution of problems $\left(P_{\lambda}\right)_{ \pm}$if it belongs to the Sobolev space $W_{0}^{1, p}(\Omega)$ and such that

i) $u \geq 0$ a.e. on $\Omega$ and $u>0$ on a subset of $\Omega$ with positive measure;

ii) for all $\varphi \in W_{0}^{1, p}(\Omega)$ the following identity holds

$$
\int_{\Omega}|\nabla u|^{p-2} \nabla u \nabla \varphi d x=\int_{\Omega}\left(\lambda k(x) u^{q} \pm h(x) u^{\sigma}\right) \varphi d x .
$$


This kind of problems with convex and concave nonlinearities have been extensively studied and plays a central role in modern mathematical sciences, in the theory of heat conduction in electrically conduction materials, in the study of non-Newtonian fluids (see: Allegretto-Huang 1], Ambrosetti-Brezis-Cerami [2], Brezis-Nirenberg [3], Guo-Zhang 9], Figueiredo-Gossez-Ubilla [8] with their references). The basic work in our direction is the article [9] where Guo and Zhang have been considered the Dirichled problem

$$
-\Delta_{p} u=\lambda u^{q}+u^{\sigma} \text { if } x \in \Omega, u>0 \text { if } x \in \Omega, u=0 \text { if } x \in \partial \Omega,
$$

where $\lambda$ is a positive parameter, $\Omega \subset \mathbb{R}^{N}(N \geq 3)$ is a bounded domain with smooth boundary, $0<q<p-1<\sigma<p^{*}-1$ inequality in which $p^{*}$ represents for the Sobolev conjugate exponent of $p$, namely $p^{*}:=N p /(N-p)$ if $1<p<N$ and $p^{*}:=\infty$ for $p \geq N$. We mention that in the work [9] the authors have been extended the results of Brezis and Nirenberg [3] obtained in the case $p=2$.

Our main goal is to extend the results obtained in [9] to the more general problems $\left(P_{\lambda}\right)_{ \pm}$.

The p-laplacian operator arises naturally in various contexts of physics, for instance, in nonNewtonian fluid theory, the quantity $p$ is a characteristic of the medium. The case $1<p<2$ corresponds to pseudoplastics fluids and $p>2$ arises in the consideration of dilatant fluids.

The main results are as follows:

Theorem 1.1. Let $p>1$. For all $0<q<p-1<\sigma<p^{*}-1$ there exists a positive number $\lambda^{*}$ such that for $\lambda \in\left(0, \lambda^{*}\right)$ the problem $\left(P_{\lambda}\right)_{+}$has a minimal solution $u(\lambda)$ which is increasing with respect to $\lambda$. If $\lambda=\lambda^{*}$ the problem $\left(P_{\lambda}\right)_{+}$has a solution. Moreover, problem $\left(P_{\lambda}\right)_{+}$does not have any solution if $\lambda>\lambda^{*}$.

Theorem 1.2. Suppose $0<q<p-1<\sigma<p^{*}-1$. Then there exists a positive number $\lambda^{*}$ such that the problem $\left(P_{\lambda}\right)_{-}$has at least one solution for $\lambda>\lambda^{*}$. Moreover, the problem $\left(P_{\lambda}\right)_{-}$does not have any solution for $\lambda<\lambda^{*}$.

Before we prove the main theorems, we need some additional results.

\section{Preliminary results}

The next result describes a regularity near the boundary for weak solutions to $\left(\left(P_{\lambda}\right)_{ \pm}\right)$and is developed by Lieberman in more general form than one presented here. For the interior regularity we advise the work of Tolksdorf [17] and DiBenedetto [7].

Lemma 2.1. (in [12] ) Let $\beta, \Lambda, M_{0}$ be positive constants with $\beta \leq 1$ and let $\Omega$ be a bounded domain in $\mathbb{R}^{N}$ with $C^{1, \beta}$ boundary. Suppose $b(x, r)$ satisfies the condition $|b(x, r)| \leq \Lambda$ for all $(x, r)$ in $\partial \Omega \times\left[-M_{0}, M_{0}\right]$. If $u$ is a bounded weak solution of the problem

$$
\Delta_{p} u+b(x, u)=0 \text { for } x \in \Omega, u=0 \text { on } \partial \Omega
$$

with $|u| \leq M_{0}$ in $\Omega$, then there is a positive constant $\alpha:=\alpha(\alpha, \Lambda, N)$ such that $u$ is in $C^{1, \alpha}(\bar{\Omega})$. Moreover $|u|_{1+\alpha} \leq C\left(\alpha, \Lambda, M_{0}, N, \Omega\right)$. 
We use in the proof the strong maximum principle of Vazquez.

Lemma 2.2. (see [18]) Let $\Omega$ be a domain in $\mathbb{R}^{N}(N \geq 1)$ and $u \in C^{1}(\Omega)$ such that $\Delta_{p} u \in L_{\text {loc }}^{2}(\Omega)$, $u \geq 0$ a.e. in $\Omega, u \neq 0, \Delta_{p} u \leq \beta(u)$ a.e. in $\Omega$ with $\beta:[0, \infty) \rightarrow \mathbb{R}$ continuous, non-decreasing, $\beta(0)=0$ and either $\beta(s)=0$ for some $s>0$ or $\beta(s)>0$ for all $s>0$ but

$$
\int_{0}^{1}(j(S))^{-1 / p} d S=\infty \text { where } j(S)=\int_{0}^{S} \beta(t) d t .
$$

Then if $u$ does not vanish identically on $\Omega$ it is positive everywhere in $\Omega$.

The following lemma has been obtained in Sakaguchi.

Lemma 2.3. (see [15]) Let $\Omega \subset \mathbb{R}^{N}(N \geq 2)$ is a bounded domain with smooth boundary and let $u \in C^{1}(\bar{\Omega}) \cap W_{0}^{1, p}(\Omega)$ satisfy:

$$
\begin{aligned}
-\Delta_{p} u & \geq 0 \text { in } \Omega(\text { in the weak sense }) \\
u & >0 \text { in } \Omega \text { and } u=0 \text { on } \partial \Omega .
\end{aligned}
$$

Then $\partial u / \partial n<0$ on $\partial \Omega$ where $n$ denotes the unit exterior normal vector to $\partial \Omega$.

The following comparison principle is proved in [15] (or consult some ideas of the proof in [16, Lemma 3.1.]).

Lemma 2.4. Let $\Omega \subset \mathbb{R}^{N}(N \geq 2)$ is a bounded domain with smooth boundary and let $u, v \in$ $W^{1, p}(\Omega)$ satisfy $-\Delta_{p} u \leq-\Delta_{p} v$ for $x \in \Omega$, in the weak sense. If $u \leq v$ on $\partial \Omega$ then $u \leq v$ in $\Omega$.

We prove Theorem 1.1 also by the method of sub- and super-solutions. To describe this method we introduce the problem

$$
-\Delta_{p} u=\lambda k(x) u^{q}+h(x) u^{\sigma} \text { for } x \in \Omega, u=0 \text { on } \partial \Omega,
$$

where $\Omega, \lambda, k, q, h$ and $\sigma$ are as above. We define $\underline{u} \in W_{0}^{1, p}(\Omega) \cap L^{\infty}(\Omega)$ to be a sub-solution of (2.1) if

$$
\begin{aligned}
-\Delta_{p} \underline{u} & \leq \lambda k(x) \underline{u}^{q}+h(x) \underline{u}^{\sigma} x \in \Omega, \text { (in the weak sense) } \\
\underline{u} & =0
\end{aligned}
$$

and $\bar{u} \in W_{0}^{1, p}(\Omega) \cap L^{\infty}(\Omega)$ to be a super-solution of (2.1) if

$$
\begin{aligned}
-\Delta_{p} \bar{u} & \geq \lambda k(x) \bar{u}^{q}+h(x) \bar{u}^{\sigma} x \in \Omega, \text { (in the weak sense) } \\
\bar{u} & =0 .
\end{aligned}
$$

Then the following result holds:

Lemma 2.5. (see [5]) Suppose there exist a sub-solution $\underline{u}$ and a super-solution $\bar{u}$ of (2.1) in the above sense and that $\underline{u} \leq \bar{u}$. Then there exists a bounded weak solution $u$ of the problem (2.1) such that $\underline{u} \leq u \leq \bar{u}$. 
We finally recall the following Picone's result for the p-Laplacian developed by Allegretto and Huang.

Lemma 2.6. (see [1]) Let $v>0, u \geq 0$ be differentiable. Denote

$$
R(u, v)=|\nabla u|^{p}-\nabla\left(\frac{u^{p}}{v^{p-1}}\right)|\nabla v|^{p-2} \nabla v .
$$

Then $R(u, v) \geq 0$ and $R(u, v)=0$ a.e. $\Omega$ if and only if $\nabla(u / v)=0$ a.e. $\Omega$, i.e. $u=k v$ for some constant $k$ in each component of $\Omega$, where $\Omega$ is bounded or unbounded, or the whole space $\mathbb{R}^{N}$.

\section{Proof of the Theorem 1.1}

Firstly, we prove that there exists $\lambda_{0}>0$ such that for all $\lambda \in\left(0, \lambda_{0}\right]$ the problem $\left(P_{\lambda}\right)_{+}$has a solution. The argument relies on constructing a sub- and a super-solution with the properties from Lemma 2.5. In order to find a sub-solution, consider the problem

$$
-\Delta_{p} u=\lambda k(x) u^{q} \text { if } x \in \Omega, u>0 \text { in } \Omega, u=0 \text { on } \partial \Omega .
$$

Then, by [6], problem (3.1) has a unique positive solution $w \in W_{0}^{1, p}(\Omega) \cap L^{\infty}(\Omega)$ with $\partial w / \partial n<0$ on $\partial \Omega$. It is not difficult to prove that the function $\underline{u}:=\varepsilon^{1 /(p-1)} w$ is a sub-solution of problem $\left(P_{\lambda}\right)_{+}$provided that $\varepsilon>0$ is small enough. For this, it suffices to observe that

$$
\varepsilon \lambda k(x) w^{q} \leq \lambda k(x) \varepsilon^{q /(p-1)} w^{q}+h(x) \varepsilon^{\sigma /(p-1)} w^{\sigma} \text { in } \Omega
$$

which is true for all $\varepsilon \in(0,1)$. Let $v \in W_{0}^{1, p}(\Omega) \cap L^{\infty}(\Omega)$ be the positive solution of

$$
\left\{\begin{array}{c}
-\Delta_{p} v=1 \text { in } \Omega \\
v=0 \text { on } \partial \Omega .
\end{array}\right.
$$

which exists and is unique from [10, Lemma 2.1.]. We prove that if $\lambda>0$ is small enough then there is $M>0$ such that $\bar{u}=M^{1 /(p-1)} v$ is a super-solution of $\left(P_{\lambda}\right)_{+}$. Therefore it suffices to show that

$$
M \geq \lambda k(x)\left[M^{1 /(p-1)} v\right]^{q}+h(x)\left[M^{1 /(p-1)} v\right]^{\sigma} .
$$

In the next, we use some notations

$$
A=\|k\|_{L^{\infty}} \cdot\|v\|_{L^{\infty}}^{q} \text { and } B=\|h\|_{L^{\infty}} \cdot\|v\|_{L^{\infty}}^{\sigma} .
$$

Thus by (3.2), it is enough to show that there is $M>0$ such that

$$
M \geq \lambda A M^{q /(p-1)}+B M^{\sigma /(p-1)}
$$

that is equivalent to

$$
1 \geq \lambda A M^{(q-p+1) /(p-1)}+B M^{(\sigma-p+1) /(p-1)} .
$$

Consider the following mapping $(0, \infty) \ni t \rightarrow \lambda A t^{(q-p+1) /(p-1)}+B t^{(\sigma-p+1) /(p-1)}$. We also note that this function reaches its minimum value in $t=C \lambda^{(p-1) /(\sigma-q)}$, where

$$
C=\left[A B^{-1}(p-1-q)(\sigma-p+1)^{-1}\right]^{(p-1) /(\sigma-q)} .
$$


Moreover, the global minimum of this mapping is

$$
\left[\left(A C^{(q-p+1) /(p-1)}+B C^{(\sigma-p+1) /(p-1)}\right)\right] \lambda^{(\sigma-p+1) /(\sigma-p)} .
$$

This show that condition (3.3) is fulfilled for all $\lambda \in\left(0, \lambda_{0}\right]$ and $M=C \lambda^{(p-1) /(\sigma-q)}$, where $\lambda_{0}$ satisfies

$$
\left[\left(A C^{(q-p+1) /(p-1)}+B C^{(\sigma-p+1) /(p-1)}\right)\right] \lambda_{0}^{(\sigma-p+1) /(\sigma-p)}=1 .
$$

Taking $\varepsilon>0$ possibly smaller, we also note that the comparison principle announced in Lemma 2.4 implies $\varepsilon^{1 /(p-1)} w \leq M^{1 /(p-1)} v$. Thus, by Lemma 2.5 the problem $\left(P_{\lambda}\right)_{+}$has at least one solution $u_{\lambda}$. Therefore, this solution is a critical point of the functional

$$
u \longrightarrow \frac{1}{p} \int_{\Omega}|\nabla u|^{p} d x-\frac{\lambda}{q+1} \int_{\Omega} k(x)|u|^{q+1} d x-\frac{1}{\sigma+1} \int_{\Omega} h(x)|u|^{\sigma+1} d x
$$

in the closed convex set $\left\{u \in W_{0}^{1, p} \mid \varepsilon^{1 /(p-1)} w \leq u \leq M^{1 /(p-1)} v\right\}$.

By choosing

$$
\lambda^{*}=\sup \left\{\lambda>0 \mid \text { problem }\left(P_{\lambda}\right)_{+} \text {has a solution }\right\},
$$

we have from the definition of $\lambda^{*}$ that problem $\left(P_{\lambda}\right)_{+}$does not have any solution if $\lambda>\lambda^{*}$. In what follows we claim that $\lambda^{*}$ is finite. Denote

$$
m:=\min \left\{\text { ess } \inf _{x \in \Omega} k(x) \text {, ess } \inf _{x \in \Omega} h(x)\right\} .
$$

Clearly, $m>0$. Let $\lambda^{\prime}>0$ be such that

$$
m\left(\lambda^{\prime} t^{q-p+1}+t^{\sigma-p+1}\right)>\lambda_{1} \text { for all } t \geq 0
$$

where $\lambda_{1}$ stands for the first eigenvalue of $\left(-\Delta_{p}\right)$ in $W_{0}^{1, p}(\Omega)$. Denote by $\varphi_{1}$ an eigenfunction of the p-Laplacian operator corresponding to $\lambda_{1}$. Then $\varphi_{1} \in C^{1, \alpha}(\bar{\Omega})$ and $\varphi_{1}>0$ in $\Omega$ as a consequence of the strong maximum principle of Vazquez (Lemma 2.2). We apply Picone's result, Lemma 2.6, to the function $\varphi_{1}$ and $u_{\lambda}$. We drop the parameter $\lambda$ in the function $u_{\lambda}$ and denote $u:=u_{\lambda}$. Observe that $\frac{\varphi_{1}^{p}}{u^{p-1}}$ belongs to $W_{0}^{1, p}(\Omega)$ since $u$ is positive in $\Omega$ and has nonzero outward derivative on the boundary because of the Hopf Lemma 2.3. Then for all $\lambda>\lambda^{\prime}$ we have

$$
\begin{aligned}
0 & \leq \int_{\Omega}\left|\nabla \varphi_{1}\right|^{p} d x-\int_{\Omega} \nabla\left(\frac{\varphi_{1}^{p}}{u^{p-1}}\right)|\nabla u|^{p-2} \nabla u d x \\
& =\int_{\Omega}\left|\nabla \varphi_{1}\right|^{p} d x-\int_{\Omega} \frac{\varphi_{1}^{p}}{u^{p-1}} \Delta_{p} u d x \\
& =\int_{\Omega}\left|\nabla \varphi_{1}\right|^{p} d x-\int_{\Omega} \frac{\varphi_{1}^{p}}{u^{p-1}}\left(\lambda k(x) u^{q}+h(x) u^{\sigma}\right) d x \\
& <\int_{\Omega} \lambda_{1} \varphi_{1}^{p} d x-\int_{\Omega} m\left[\lambda k(x) u^{q-p+1}+h(x) u^{\sigma-p+1}\right] \varphi_{1}^{p} d x \\
& <\int_{\Omega} \lambda_{1} \varphi_{1}^{p} d x-\int_{\Omega} m\left[\lambda^{\prime} u^{q-p+1}+u^{\sigma-p+1}\right] \varphi_{1}^{p} d x \\
& =\int_{\Omega}\left[\lambda_{1}-m\left(\lambda^{\prime} u^{q-p+1}+u^{\sigma-p+1}\right)\right] \varphi_{1}^{p} d x<0 .
\end{aligned}
$$

Thus we get a desired contradiction. As a conclusion we obtain the following result $\lambda^{*} \leq \lambda^{\prime}<\infty$ which proves our claim. Let as now prove that $u_{\lambda}$ is a minimal solution of the problem $\left(P_{\lambda}\right)_{+}$. 
By the definition of $\lambda^{*}$ there exists $\bar{\lambda}<\lambda$ such that $\bar{\lambda}<\lambda^{*}$ and $\left(P_{\bar{\lambda}}\right)_{+}$has a positive solution $u_{\bar{\lambda}}$. The rest of the argument is based on the standard monotone iteration. Consider the sequence $\left(u_{n}\right)_{n \geq 0}$ defined by $u_{0}=w$ (where $w$ is the unique solution of (3.1)) and $u_{n}$ the solution of the problem

$$
\begin{aligned}
-\Delta_{p} u_{n} & =\lambda k(x) u_{n-1}^{q}+h(x) u_{n-1}^{\sigma}, \text { if } x \in \Omega \\
u_{n}(x) & >0, \text { if } x \in \Omega \\
u_{n}(x) & =0, \text { if } x \in \partial \Omega
\end{aligned}
$$

which exists and is unique from the results in [11] (see also arguments in [9]). By using the comparison principle, it is not hard to show that

$$
u_{0}=w \leq u_{1} \leq \ldots \leq u_{n} \leq u_{n+1} \leq u_{\bar{\lambda}} \text { in } \Omega .
$$

In fact, it follows again by the above cited comparison principle applied to the problem

$$
\begin{aligned}
-\Delta_{p} u_{0} & =\lambda k(x) u_{0}^{q} \leq \lambda k(x) u_{0}^{q}+h(x) u_{0}^{\sigma}=-\Delta_{p} u_{1} \text { in } \Omega, \\
u_{0} & =u_{1}=0 \text { on } \partial \Omega
\end{aligned}
$$

that $u_{0} \leq u_{1}$ in $\Omega$. Similarly, one can show by using the same Lemma 2.4 that $u_{1} \leq u_{2}$ in $\Omega$. In particular, for all $x \in \Omega$ the sequence $\left(u_{n}\right)_{n \geq 0}$ is a nondecreasing sequence which is bounded and therefore $u_{n} \leq U$ for any positive solution $U$ of $\left(P_{\lambda}\right)_{+}$. Using the relation (3.5), the decay property of $u_{\bar{\lambda}}$ and a standard diagonalization procedure we get a subsequence converging to a solution $u_{\lambda}$ of $\left(P_{\lambda}\right)_{+}$, satisfying $u_{\lambda} \leq u_{\bar{\lambda}}$ and $u_{\lambda} \leq U$ for any arbitrary solution $U$ of problem $\left(P_{\lambda}\right)_{+}$. The conclusion then follow. At this stage it is easy to deduce that the mapping $u_{\lambda}$ is increasing with respect to $\lambda$. We consider $u_{\lambda_{1}}, u_{\lambda_{2}}$ with $0<\lambda_{1}<\lambda_{2}<\lambda^{*}$. Since

$$
-\Delta_{p} u_{\lambda_{2}}=\lambda_{2} k(x) u_{\lambda_{2}}^{q}+h(x) u_{\lambda_{2}}^{\sigma}>\lambda_{1} k(x) u_{\lambda_{2}}^{q}+h(x) u_{\lambda_{2}}^{\sigma}
$$

then $u_{\lambda_{2}}$ is a super-solution of problem $\left(P_{\lambda_{1}}\right)_{+}$. The argument used above may be used to construct a sequence $\left(u_{n}\right)_{n \geq 0}$ such that $0<u_{n-1}<u_{n}<u_{\lambda_{2}}$ converging to a solution $U$ of $\left(P_{\lambda_{1}}\right)_{+}$with $U<u_{\lambda_{2}}$ and therefore $u_{\lambda_{1}} \leq U<u_{\lambda_{2}}$ by the minimality of $u_{\lambda_{1}}$. This proves our claim.

It remain to show that problem $\left(P_{\lambda}\right)_{+}$has a solution if $\lambda=\lambda^{*}$. For this purpose it is enough to prove that

$$
u_{\lambda} \text { is bounded in } W_{0}^{1, p}(\Omega) \text { as } \lambda \rightarrow \lambda^{*} .
$$

Thus, by passing to a suitable subsequence if necessary, we may assume

$$
u_{\lambda} \rightarrow u^{*} \text { in } W_{0}^{1, p}(\Omega) \text { as } \lambda \rightarrow \lambda^{*},
$$

which implies that $u^{*}$ is a weak solution of $\left(P_{\lambda}\right)_{+}$provided that $\lambda=\lambda^{*}$. Moreover since the mapping $\lambda \rightarrow u_{\lambda}$ is increasing, it follows that $u^{*} \geq 0$ a.e. on $\Omega$ and $u^{*}>0$ on a subset of $\Omega$ with positive measure. As we mentioned, it is often advantageous to work with $u$ instead of $u_{\lambda}$. A key ingredient of the proof is that all solutions $u$ have negative energy. More precisely, if $E: W_{0}^{1, p}(\Omega) \rightarrow \mathbb{R}$ is defined by

$$
E(u)=\frac{1}{p} \int_{\Omega}|\nabla u|^{p} d x-\frac{\lambda}{q+1} \int_{\Omega} k(x)|u|^{q+1} d x-\frac{1}{\sigma+1} \int_{\Omega} h(x)|u|^{\sigma+1} d x
$$


then

$$
E(u)<0 \text { for all } \lambda \in\left(0, \lambda^{*}\right) \text {. }
$$

We do it in the following steps:

Step 1) the solution $u$ satisfies

$$
\int_{\Omega}\left\{|\nabla u|^{p}-[\lambda q /(p-1)] k(x) u^{q+1}+[\sigma /(p-1)] h(x) u^{\sigma+1}\right\} d x \geq 0 .
$$

This follows by the same arguments from [9, Lemma 3.7.].

Step 2) Since $u$ is a solution of $\left(P_{\lambda}\right)_{+}$we have

$$
\int_{\Omega}|\nabla u|^{p} d x=\int_{\Omega} \lambda k(x) u^{q+1} d x+\int_{\Omega} h(x) u^{\sigma+1} d x .
$$

Plugging relation (3.8) into (3.9) we have

$$
\lambda(p-1-q) \int_{\Omega} k(x) u^{q+1} d x \geq(\sigma+1-p) \int_{\Omega} h(x) u^{\sigma+1} d x
$$

In particular, it follows from these two latest relations that

$$
\begin{aligned}
E(u) & =\lambda\left(\frac{1}{p}-\frac{1}{q+1}\right) \int_{\Omega} k(x) u^{q+1} d x+\left(\frac{1}{p}-\frac{1}{\sigma+1}\right) \int_{\Omega} h(x) u^{\sigma+1} d x \\
& =-\lambda \frac{p-1-q}{p(q+1)} \int_{\Omega} k(x) u^{q+1} d x+\frac{\sigma+1-p}{p(\sigma+1)} \int_{\Omega} h(x) u^{\sigma+1} d x \\
& \leq-\lambda \frac{p-1-q}{p(q+1)} \int_{\Omega} k(x) u^{q+1} d x+\lambda \frac{p-1-q}{p(\sigma+1)} \int_{\Omega} h(x) u^{\sigma+1} d x \leq 0 .
\end{aligned}
$$

Thus, by combining (3.7) and (3.8), sobolev embedings, and using the fact that $k, h \in L^{\infty}(\Omega)$ it follows

$$
\sup \left\{\left\|u_{\lambda}\right\|_{W_{0}^{1, p}(\Omega)} \mid \lambda<\lambda^{*}\right\}<\infty
$$

and so (3.6) is finished. This complete the proof of Theorem 1.1.

\section{Proof of the Theorem 1.2}

The study of existence of solutions to problem $\left(P_{\lambda}\right)_{-}$is done by looking for critical points of the functional $F_{\lambda}: W_{0}^{1, p}(\Omega) \rightarrow \mathbb{R}$ defined by

$$
F_{\lambda}(u)=\frac{1}{p} \int_{\Omega}|\nabla u|^{p} d x-\frac{\lambda}{q+1} \int_{\Omega} k(x)|u|^{q+1} d x+\frac{1}{\sigma+1} \int_{\Omega} h(x)|u|^{\sigma+1} d x .
$$

In the next we adopt the following notations

$$
\|u\|:=\left(\int_{\Omega}|\nabla u|^{p} d x\right)^{1 / p},\|u\|_{q+1}:=\left(\int_{\Omega}|u|^{q+1} d x\right)^{1 /(q+1)},\|u\|_{\sigma+1}:=\left(\int_{\Omega}|u|^{\sigma+1} d x\right)^{1 /(\sigma+1)} .
$$

We prove that $F_{\lambda}$ is coercive. In order to verify this claim, we first observe that

$$
F_{\lambda}(u) \geq \frac{1}{p}\|u\|^{p}-C_{1}\|u\|_{q+1}^{q+1}+C_{2}\|u\|_{\sigma+1}^{\sigma+1}
$$


where

$$
C_{1}=\frac{\lambda}{q+1}\|k\|_{L^{\infty}} \text { and } C_{2}=\frac{1}{\sigma+1} \text { ess } \inf _{x \in \Omega} h(x)
$$

are positive constants. Since $q<\sigma$, a simple calculation shows that the mapping

$$
(0, \infty) \ni t \rightarrow C_{1} t^{q+1}-C_{2} t^{\sigma+1}
$$

attains its global minimum $m<0$ at

$$
t=\left[\frac{C_{2}(q+1)}{C_{1}(\sigma+1)}\right]^{1 /(\sigma-q)} .
$$

So we conclude that

$$
F_{\lambda}(u) \geq \frac{1}{p}\|u\|^{p}+m
$$

and hence $F_{\lambda}(u) \rightarrow \infty$ as $\|u\| \rightarrow \infty$ whish finished the proof that $F_{\lambda}$ is coercive. Let $\left(u_{n}\right)_{n \geq 0}$ be a minimizing sequence of $F_{\lambda}$ in $W_{0}^{1, p}(\Omega)$. The coercivity of $F_{\lambda}$ implies the boundedness of $u_{n}$ in $W_{0}^{1, p}(\Omega)$. Then, up to a subsequence if necessary, we may assume that there exists $u$ in $W_{0}^{1, p}(\Omega)$ non-negative such that $u_{n} \stackrel{n \rightarrow \infty}{\rightarrow} u$ weakly in $W_{0}^{1, p}(\Omega)$. We remark that the function $u$ can be non-negative due to $F_{\lambda}(u)=F_{\lambda}(|u|)$. Standard arguments based on the lower semi-continuity of the energy functional show that $u$ is a global minimizer of $F_{\lambda}$ and therefore is a solution in the sense of distributions of $\left(P_{\lambda}\right)_{-}$.

In what follows we claim that the weak limit $u$ is a non-negative weak solution of problem $\left(P_{\lambda}\right)_{-}$if $\lambda>0$ is large enough. We first observe that $F_{\lambda}(0)=0$. So, in order to prove that the non-negative solution is non-trivial, it suffices to prove that there exists $\Lambda>0$ such that

$$
\inf _{u \in W_{0}^{1, p}(\Omega)} F_{\lambda}(u)<0 \text { for all } \lambda>\Lambda
$$

For this purpose we consider the variational problem with constraints,

$\Lambda=\inf \left\{\frac{1}{p} \int_{\Omega}|\nabla v|^{p} d x+\frac{1}{\sigma+1} \int_{\Omega} h(x)|v|^{\sigma+1} d x \mid v \in W_{0}^{1, p}(\Omega)\right.$ and $\left.\frac{1}{q+1} \int_{\Omega} k(x)|v|^{q+1} d x=1\right\}$.

Let $\left(v_{n}\right)_{n \geq 0}$ be an arbitrary minimizing sequence for this problem. Then $v_{n}$ is bounded, hence we can assume that it weakly converges to some $v \in W_{0}^{1, p}(\Omega)$ with

$$
\frac{1}{q+1} \int_{\Omega} k(x)|v|^{q+1} d x=1 \text { and } \Lambda=\frac{1}{p} \int_{\Omega}|\nabla v|^{p} d x+\frac{1}{\sigma+1} \int_{\Omega} h(x)|v|^{\sigma+1} d x .
$$

Thus

$$
F_{\lambda}(v)=\Lambda-\lambda \text { for all } \lambda>\Lambda
$$

Set

$$
\lambda^{*}:=\inf \left\{\lambda>0 \mid \text { problem }\left(P_{\lambda}\right)_{-} \text {admits a nontrivial weak solution }\right\} \geq 0 .
$$

The above remarks show that $\Lambda \geq \lambda^{*}$ and that problem $\left(P_{\lambda}\right)_{-}$has a solution for all $\lambda \geq \Lambda$. We now argue that problem $\left(P_{\lambda}\right)_{-}$has a solution for all $\lambda>\lambda^{*}$. Fixed $\lambda>\lambda^{*}$, by the definition of $\lambda^{*}$, we can take $\mu \in\left(\lambda^{*}, \lambda\right)$ such that $F_{\mu}$ has a nontrivial critical point $u_{\mu} \in W_{0}^{1, p}(\Omega)$. Since $\mu<\lambda$, 
it follows that $u_{\mu}$ is a sub-solution of problem $\left(P_{\lambda}\right)_{-}$. We now want to construct a super-solution that dominates $u_{\mu}$. For this purpose we consider the constrained minimization problem

$$
\inf \left\{F_{\lambda}(v), v \in W_{0}^{1, p}(\Omega) \text { and } v \geq u_{\mu}\right\} .
$$

From the previous arguments, used to treat (4.1) follows that problem (4.2) has a solution $u_{\lambda}>u_{\mu}$. Moreover, $u_{\lambda}$ is a solution of problem $\left(P_{\lambda}\right)_{-}$for all $\lambda>\lambda^{*}$. With the arguments developed in [9] we deduce that problem $\left(P_{\lambda}\right)_{-}$has a solution if $\lambda=\lambda^{*}$. The same monotonicity arguments as above show that $\left(P_{\lambda}\right)_{-}$does not have any solution if $\lambda<\lambda^{*}$. Fix $\left.\lambda\right\rangle \lambda^{*}$. It remains to argue that the non-negative weak solution $u$ is, in fact, positive. Indeed, using Moser iteration, we obtain that $u \in L^{\infty}(\Omega)$. Once $u \in L^{\infty}(\Omega)$ it follows by Lemma 2.1 that $u$ is a $C^{1, \alpha}(\bar{\Omega})$ solution of problem $\left(P_{\lambda}\right)_{-}$provided for some $\alpha$. Invoking the nonlinear strong maximum principle of Vazquez (Lemma 2.2), since $u$ is a non-negative smooth weak solution of the differential inequality

$$
-\Delta_{p} u+h(x) u^{\sigma} \geq 0 \text { in } \Omega,
$$

we deduce that $u$ is positive everywhere in $\Omega$. The proof of Theorem 1.2 is completed.

The extension of the above results to all space $\mathbb{R}^{N}$ or to the nonlinearities depending on the gradient $\nabla u$ requires some further nontrivial modifications and will be considered in a future work. We anticipate that the methods and concepts here can be extended to systems or when in discussion are more general linear/non-linear operators as well.

\section{References}

[1] W. Allegretto and Y-X. Huang, A Piccone's identity for the p-Laplacian and applications, Nonlinear Analysis: Theory, Methods \& Applications, Volume 32, No. 7, Pages 819-830, 1998.

[2] A. Ambrosetti, H. Brezis, G. Cerami, Combined effects of concave and convex nonlinearities in some elliptic problems, Journal of Functional Analysis, Volume 122, No. 2, Pages 519-543, 2009.

[3] H. Brezis and L. Nirenberg, $H^{1}$ versus $C^{1}$ local minimizers, C. R. Acad. Sci. Paris Ser. I Math., Volume 317, No. 5, Pages 465-472, 1993.

[4] H. Brezis and L. Nirenberg, Positive solutions of nonlinear elliptic equations involving critical Sobolev exponents, Communications on Pure and Applied Mathematics, Volume 36, No. 4, Pages 437-477, 1983.

[5] X. Cabre and M. Sanchon, Semi-stable and extremal solutions of reaction equations involving the p-Laplacian, Communications in Pure and Applied Analysis, Volume 6, Pages 43-67, 2007.

[6] J. I. Diaz and J. E. Saà, Existence et unicite de solutions positives pour certaines equations elliptiques quasilineaires, CRAS 305 Serie I, Pages 521-524, 1987.

[7] E. DiBenedetto, $C^{1, \alpha}$ - local regularity of weak solutions of degenerate elliptic equations, Nonlinear Analysis: Theory, Methods \& Applications, Volume 7, Issue 8, Pages 827-850, 1983. 
[8] D. G. de Figueiredo, J.-P. Gossez and P. Ubilla, Local "superlinearity" and "sublinearity" for the p-Laplacian, Journal of Functional Analysis, Volume 257, Pages 721-752, 2009.

[9] Z. Guo and Z. Zhang, $W^{1, p}$ versus $C^{1}$ local minimizers and multiplicity results for quasilinear elliptic equations, Journal of Mathematical Analysis and Applications, Volume 286, Issue 1, Pages 32-50, 1 October 2003.

[10] Z. Guo and J.R.L. Webb, Uniqueness of positive solutions for quasilinear elliptic equations when a parameter is large, Proceedings of the Royal Society of Edinburgh, Volume 124A, Pages 189-198, 1994.

[11] J. Leray and J.L. Lions, Quelques resultats de Visik sur les problemes elliptiques nonlineaires par les methodes de Minty-Browder, Bulletin de la Société Mathématique de France, Volume 93, Pages 97-107, 1965.

[12] G. M. Lieberman, Boundary regularity for solutions of degenerate elliptic equations, Nonlinear Analysis: Theory, Methods \& Applications, Volume 12, Issue 11, Pages 1203-1219, November 1988.

[13] K Perera and R. Shivaji, Positive solutions of multiparameter semipositone p-Laplacian problems, Journal of Mathematical Analysis and Applications, Volume 338, No. 2, Pages 13971400, 2008.

[14] V. Radulescu and D. Repovs, Combined effects in nonlinear problems arising in the study of anisotropic continuous media, Nonlinear Analysis: Theory, Methods \& Applications, (2011), doi:10.1016/j.na.2011.01.037

[15] S. Sakaguchi, Concavity properties of solutions to some degenerate quasilinear elliptic Dirichlet problems, Annali della Scuola Normale Superiore di Pisa, Classe di Scienze 4e sèrie, tome 14, no. 3, Pages 403-421, 1987.

[16] P. Tolksdorf, On the Dirichlet problem for quasilinear equations in domains with conical boundary points, Communications in Partial Differential Equations, Volume 8, Issue7, Pages 773-817, 1983.

[17] P. Tolksdorf, Regularity for a more general class of quasilinear elliptic equations, Journal of Differential Equations, Volume 51, Issue 1, Pages 126-150, January 1984.

[18] J.L. Vázquez, A strong maximum principle for some quasilinear elliptic equations, Applied Mathematics and Optimization, Volume 12, Pages 191-202, 1984. 